Cite as: Lahousse L. Amazing pleiotropic effects of azithromycin. Breathe 2018; 14: 336-337.

\title{
Amazing pleiotropic effects of azithromycin
}

\section{Journal club}

\section{Commentary on:}

Gibson PG, et al. Effect of azithromycin on asthma exacerbations and quality of life in adults with persistent uncontrolled asthma (AMAZES): a randomised, double-blind, placebo-controlled trial. Lancet 2017; 390: 659-668.

The AMAZES study was a large randomised, doubleblind, placebo-controlled trial of azithromycin $500 \mathrm{mg}$ given three times a week for 48 weeks in adults with persistent uncontrolled asthma to decrease asthma exacerbations and improve asthmarelated quality of life [1]. The study was nationally funded in Australia and included 420 patients around the age of 60 years who were predominantly female (61\%). Azithromycin reduced moderate-to-severe asthma exacerbations compared with placebo and significantly improved asthma-related quality of life. Only diarrhoea was significantly more frequent in users of azithromycin compared with placebo, although patients with hearing impairment or an abnormal prolongation of the corrected QT interval were excluded from the start of the study.

The patients with asthma included in the study were persistently symptomatic despite optimised asthma treatment with inhaled corticosteroids and long-acting bronchodilators. Importantly, since azithromycin showed no benefit in current smokers with chronic obstructive pulmonary disease [2], the patients with asthma included in the study were nonsmokers (confirmed by exhaled carbon monoxide $<10$ ppm) or ex-smokers with either $\leq 10$ pack-years of smoking or without emphysema (confirmed by a carbon monoxide transfer coefficient $\geq 70 \%$ of the predicted value) [1]. Several measures were taken to favourably influence therapy adherence during the study. Only patients who were adherent (>80\%) during a 2-week run-in period were randomised. Adherence was assessed during several study visits and telephone assessments were performed three times between study visits.

The primary aim was to evaluate the effect of azithromycin on asthma exacerbations. Therefore, the study sample was sized to take into account a baseline incidence of asthma exacerbations of 0.58 per person-year and a $35 \%$ reduction in exacerbation incidence by azithromycin. Moderate exacerbations were defined as worsening of asthma symptoms that led to any temporary increase in use of inhaled corticosteroids or antibiotics, or an increase in $\beta_{2}$-agonist use for at least 2 days, or an emergency department visit (without systemic 
corticosteroids). Severe exacerbations were defined as worsening of asthma symptoms that led to a $10 \mathrm{mg}$ day $^{-1}$ (increased) systemic corticosteroid treatment for at least 3 days or an asthma-specific hospitalisation or emergency department visit requiring systemic corticosteroids. Exacerbations were registered using patient daily diaries and structured interviews during the study centre visits. The study observed that azithromycin reduced the incidence of exacerbations by $41 \%$.

The AMAZES study confirmed the previously observed benefit of macrolides on asthma symptoms and clearly contributed to the evidence for an effect on asthma exacerbations. However, potential phenotype-specific effects have not yet been fully clarified. The allergic eosinophilic phenotype is usually very corticosteroid sensitive, while the non-eosinophilic phenotype could especially benefit from therapies other than corticosteroids to control the innate immune dysfunction. While in the AZISAST study azithromycin decreased the exacerbation rate only in patients with noneosinophilic asthma [3], azithromycin reduced exacerbations in both eosinophilic asthma and noneosinophilic asthma within the AMAZES study [1]. However, the AMAZES study substantially differed from the AZISAST study in terms of phenotype definition, patient selection, duration and dosing of azithromycin. Even the primary outcome differed as the AZISAST study evaluated the effect on severe asthma exacerbations as well as on lower respiratory infections [1, 3]. In addition, sputum neutrophils did not differ between treatment groups within the AMAZES study [1].

Although macrolides are antibacterial (inhibiting bacterial protein biosynthesis) and potentially antiinflammatory (inhibiting eosinophil differentiation and accumulation of neutrophils, lymphocytes and macrophages), the AMAZES study also suggested pleiotropic antiviral effects since the benefit of azithromycin was observed on both asthma exacerbations and respiratory infections. Within the AMAZES study, azithromycin also affected lower respiratory tract infections in non-eosinophilic patients.

The clinical implication for asthma management is that azithromycin may offer relief as an add-on treatment in adult patients with persistent uncontrolled asthma during periods of increased exacerbations or respiratory infections. Before considering any additional therapy, avoidable exposures, treatable comorbidities, inhalation technique and therapy adherence to inhaled corticosteroids and long-acting $\beta_{2}$-agonists should be evaluated first. Additional evidence would help clinical practice to tailor macrolides in uncontrolled asthmatics better or to support their cost-effectiveness.

While potentially preventing substantial costs by postponing use of monoclonal antibodies and reducing side-effects of maintenance oral corticosteroids, amazing life-saving antimicrobial agents also have drawbacks. One third of the patients on azithromycin treatment within the AMAZES study suffered from diarrhoea, which was significantly higher compared with placebo. Although the sputum cultures detecting potentially pathogenic microorganisms only increased slightly from $19.6 \%$ to $20.6 \%$ at the end of treatment among both groups, the long-term effects of reallife use of maintenance macrolides on community microbial resistance requires further investigation. The risk-benefit assessment is undoubtedly different under real-life circumstances where cardiac risk assessment and therapy adherence will be less optimal, and therefore the observed benefits might be less amazing than observed within this randomised clinical trial.

\section{Conflict of interest}

L. Lahousse reports other (expert consultation) from Boehringer Ingelheim $\mathrm{GmbH}$ and Novartis, and unrestricted grants from AstraZeneca and Chiesi, outside the submitted work.

\section{References}

1. Gibson PG, Yang IA, Upham JW, et al. Effect of azithromycin on asthma exacerbations and quality of life in adults with persistent uncontrolled asthma (AMAZES): a randomised, double-blind, placebo-controlled trial. Lancet 2017; 390: 659-668.

2. Albert RK, Connett J, Bailey WC, et al. Azithromycin for prevention of exacerbations of COPD. N Engl J Med 2011; 365: 689-698.
3. Brusselle GG, Vanderstichele C, Jordens P, et al. Azithromycin for prevention of exacerbations in severe asthma (AZISAST): a multicentre randomized double-blind placebo-controlled trial. Thorax 2013; 68: 322-329. 